\title{
AUTOMATIC CONTOUR RETRIEVAL IN ANNOTATED TRUS PROSTATE IMAGES
}

\author{
Geoffroy Rivet Sabourin', Alexandra Branzan Albu', Denis Laurendeau', Luc Beaulieu ${ }^{3}$ \\ ${ }^{1}$ Dept. of ECE, Laval University (QC), ${ }^{2}$ Dept. of ECE, University of Victoria (BC), ${ }^{3}$ Hotel-Dieu Hospital, Quebec, (QC), Canada \\ grivet@gel.ulaval.ca, aalbu@ece.uvic.ca, laurend@gel.ulaval.ca, beaulieu@phy.ulaval.ca
}

\begin{abstract}
The approach proposed in this paper retrieves contours from transrectal ultrasound (TRUS) prostate images. The input images are sparsely annotated by radiologists for the purpose of brachytherapy planning and post-interventional monitoring. The theoretical contribution of the paper consists in the design of a task-oriented, bottom-up method which mimics perceptual grouping mechanisms for contour retrieval. The proposed approach is task-oriented because it embeds prior anatomical and procedural knowledge. From a practical standpoint, the proposed approach is of clinical relevance, since it allows for retrieving contours from images where the annotation is 'blended' with the image content. While new image annotation systems are able to store image content and annotations in a separate manner, many TRUS prostate databases still contain 'blended' annotations only. Our approach allows for contour retrieval and further 3D prostate modeling from such databases.
\end{abstract}

Index Terms: ultrasound imaging, image segmentation

\section{INTRODUCTION}

Recent advances in medical imaging technologies have introduced significant changes in medical practice; digital images play now a central role in diagnosis and therapy planning. In many applications, radiologists annotate images for conveying information. For instance, contours of organs can be specified on ultrasound images. While some recent annotation techniques allow for a direct separation of the annotation data from the image content, older but still clinically in use techniques 'blend' the annotation with the image content. Our paper deals with this latter case. It is believed that information retrieval from annotated 'blended' images has high applicability in creating reference image datasets and 3D prostate models from archived image databases. The importance of such reference datasets is outlined in [1].

Although humans perceive well sparsely annotated contours, it is still a challenge for a computer to achieve the same task. We propose an automatic method for extracting the contours added by radiologists on transrectal ultrasound images (TRUS) for prostate cancer therapy planning.

The medical imaging literature contains examples of algorithms proposed for the direct extraction of prostate contours from TRUS images without annotations. A major challenge is the presence of speckle noise which is characteristic to ultrasound imaging. Therefore, such algorithms usually require manual specification of seed points, such as in [2], or use manual segmentations in a training phase [3]. Other automatic methods use deformable models based on super ellipses [4] and on statistical texture matching [5]. However, automatic and semi-automatic segmentation of raw TRUS images has yet to gain clinical acceptance, mainly because radiologists require control over the segmentation results [6].

The proposed approach extracts contours annotated by radiologists for therapy planning and 3D prostate modelling. The remainder of the paper is structured as follows. Section 2 describes the proposed approach, while section 3 discusses experimental results. The last section draws conclusions and describes future work.

\section{PROPOSED APPROACH}

The proposed technique is applied to TRUS images acquired with Nucletron's FIRST ${ }^{\mathrm{TM}}$. As shown in Fig. 1, this system allows the radiologist to annotate prostate contours by specifying sparse points on each slice.
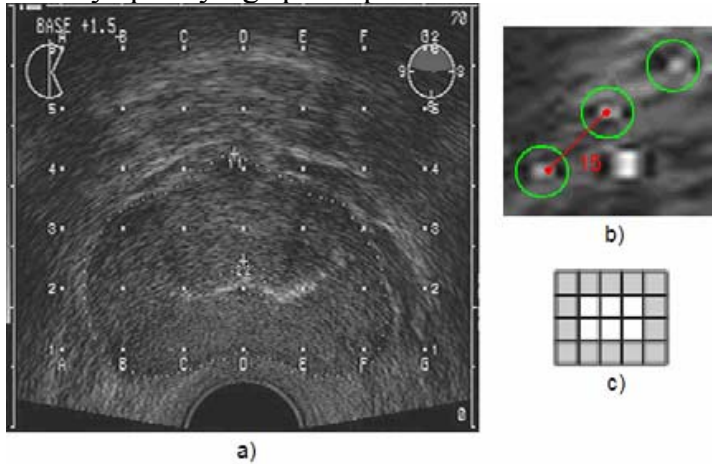

Fig. 1. a) a typical annotated TRUS image; b) zoom-in on 3 contour points (encircled) and 1 grid point; c) $5 \times 4$ convolution mask for point detection.

Perceptual grouping mechanisms in human vision perform an accurate detection of prostate contours from annotated images. However, such mechanisms are not easily reproducible by computer vision. Some reasons are the poor signal-to-noise ratio in TRUS imaging, the similar texture inside and outside the contour, as well as the presence of grid markers and various other alphanumeric symbols.

Our approach integrates anatomical information (i.e. prostate shape), as well as procedural knowledge from image acquisition and annotation into a set of basic assumptions and parameter settings. For instance, since the prostate always lies in the central part of the TRUS image, 
a rectangular region of interest (ROI) is defined by detecting vertical side lines and the semi-circular probe. The ROI center roughly approximates the prostate's centre of mass.

The proposed approach for automatic contour retrieval involves several steps. The first one detects points and groups them into chains on a slice-by-slice basis. The second step uses inter-slice similarity for recovering missed detections in step 1 and for creating new chains. The final contour is generated via a custom chain linking algorithm and Bezier interpolation. All steps are detailed below.

A. Detection and filtering of prostate contour points. The annotated prostate contour consists of a series of quasiequally spaced points typically separated by 15 pixels (see Fig. 1b); all contour points look like bright rectangular regions of $2 \times 3$ pixels. A natural solution for point detection using topological- and shape-based knowledge is template matching with a convolution mask (see Fig. 1c). The inner pattern in this mask is identical in shape and brightness to an ideal contour point, while its boundary mimics the transition to background (i.e. the TRUS image). The convolution result is binarized with non-maxima suppression. As shown in Fig. 2a, most of the detected points belong to the prostate contour. However, false detections are generated by grid points or by texture/noise; such false detections are to be removed by further filtering. The design of a 3-step filtering sequence for removing false detections is based on a priori knowledge as follows:

a) Multiple detections are false, since they are generated by grid points and other symbols. Thus, they are filtered by eliminating candidates having their nearest neighbors located within a 3 pixel radius.

b) Correct detections tend to form an ellipse. Thus, step 2 computes the distance between each candidate and the ROI center, builds a distance histogram, detects the histogram bin with the largest count and retains only the candidates populating this bin. Unfortunately, this step also eliminates some correct detections and fails to remove some erroneous candidates. Such errors occur because the ROI center (see Fig. 2b) is a raw approximation of the prostate center.

c) Adjacent points in the prostate contour are roughly at 15 pixels distance; thus, candidates with no neighbors within a 20 pixels radius are removed.

A typical result of the filtering approach is shown in Fig. $2 b$. One may notice that some false/failed detections are still present. The candidates retained after filtering are ordered using a polar representation with respect to the ROI center.
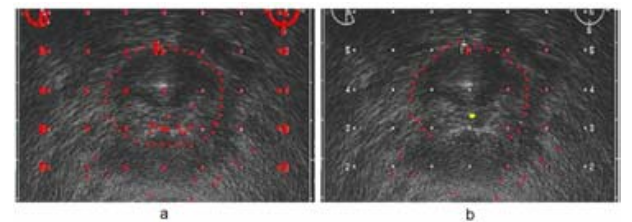

Fig. 2. a) point detections (in red) with template matching; b) filtering result (in red) and center of ROI (in yellow)
$B$. Chain generation. A chain is defined as a polygonal line composed of ordered candidates. Chains belonging to the prostate contour are expected to feature smooth curvature. The local curvature is estimated at each chain point (except extremities) by the angle defined by the current point, its successor and its predecessor (see Fig. 3a).

The iterative chain generation is described in Algorithm 1. While generating a chain, each iteration adds one point to it. This is achieved by inspecting points that are close (within $30=2 \times 15$ pixels) to the last chain point, and by selecting the point which maximizes the contour smoothness in terms of curvature (see Fig. 3b). If a minimal curvature threshold is not satisfied, then no point is added to the current chain which is considered complete. Chains with less than 3 points are eliminated, since their curvature cannot be computed. Removal of discarded candidates can be interpreted as filtering detections with a curvature criterion.
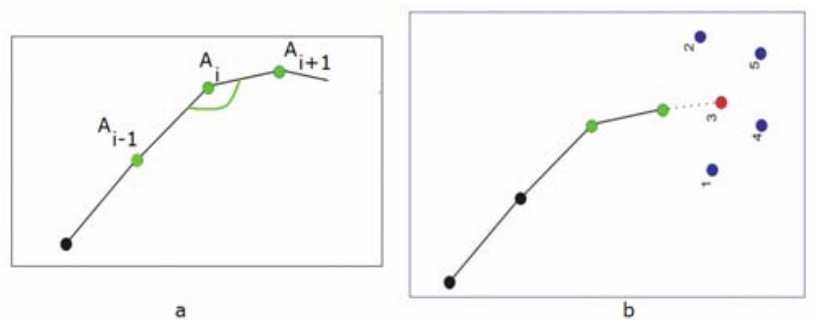

Fig. 3. a) measuring local chain curvature ; b) selection of the next chain point (red); blue points are removed

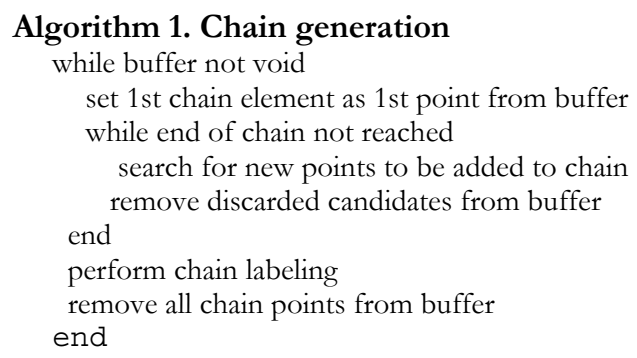

C. Chain linking. Algorithm 1 produces a series of chains that mostly belong to the prostate contour although spurious chains still persist. Thus, removal of spurious chains is integrated with chain linking in Algorithm 2. The initial reference chain must have a high probability of belonging to the prostate contour and it is chosen as the chain with maximal arc length/chain length ratio (see Fig. $4 \mathrm{~b}$ ). This choice insures an optimal orientation of the reference chain. Chain candidates for linking are chosen according to their proximity to the current reference; the closest chain from reference is the chain with the closest start point from the reference end point. Thresholding removes chains that break the curvature smoothness. Fig. $4 \mathrm{c}$ shows a synthetic example of linking chains.

The removal of spurious chains results sometimes in eliminating some valid chains; this is caused by the use of a 
fixed angular threshold (1.1 radians). The result of point detection, chain generation and chain linking on a slice-byslice basis is represented by an ordered set of chains (see Fig. 4c) which all belong to the prostate. Such chains do not form a closed contour, due to elimination of valid points during point filtering, and of valid chains during chain filtering. The retrieval of missed detections, and thus the closing of the prostate contour are achieved in step D.

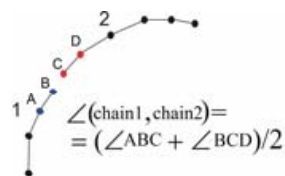

a)

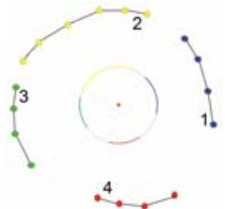

b)

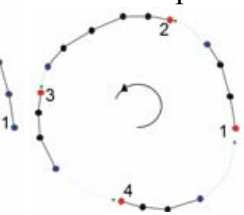

c)
Fig. 4. a) computing the angle between two chains; b) projecting 4 prostate chains onto circle of unit length at the centre of ROI; c)Ordering chains counterclockwise

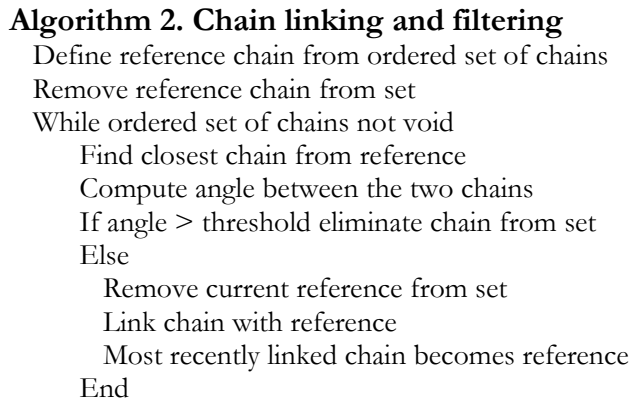

D. Additional chain retrieval using inter-slice similarity. TRUS acquisition results in a series of parallel and equidistant slices for each patient. Thus, inter-slice contour similarity can be used as a cue for retrieving chains in slice $i$ using information from slices $i+1$ and $i-1$. Extreme slices will use one neighboring slice only.

First, algorithm 3 selects chains in adjacent slices which are useful for processing the current slice. Such chains must 'cover' arcs of the unit circle which are not 'covered' by the actual set (see Fig. 6a). Selected chains in slices $i-1$ and $i+1$ are projected onto slice $i$ (see Fig. 6). Projected and actual chains may 'overlap' partially. The 'overlapping' parts of the projected chains contain redundant information and thus are removed. The remaining points in the projected chains are used as seeds for a neighborhood search of missing contour points (see Fig. 6c). New points are grouped into new chains or appended to existing chains. The final set of ordered chains (Fig. 6e) is transformed into a closed contour with Bezier interpolation (see Fig. 6f).

Algorithm 3. Chain generation with inter-slice similarity

Select relevant chains from slices $i-1, i+1$

Project these chains in $i$ and remove partial overlaps

Search missed prostate contour points in $i$ using seeds

Link newly detected points into chains using algo 1

Merge all chains in $i$ using algo 2

Close prostate contour using Bezier interpolation

\section{EXPERIMENTAL RESULTS}

The proposed approach was tested on 10 annotated TRUS patient-specific volumes. These volumes consist in parallel series of 6 to 10 slices separated by a $5 \mathrm{~mm}$ gap. The proposed approach was run without user intervention while maintaining the set of parameters constant on all data. Validation compared results on a slice-by-slice basis with reference contours obtained by manual point linking.

A validation technique able to quantify local differences between two contours was used. First, the centers of mass of each pair of corresponding 2D contours are aligned. Next, the contours are scanned simultaneously and the distance between every two points located on each contour and on the same radius is stored into a distance map (see Fig. 6a). Local differences (or defects) in the compared 2D contours correspond to local maxima in the generated distance map and are described by two parameters, namely length and depth (see Fig. 6b). The length of a defect is the length of the curved contour segment forming the defect, whereas the depth is the maximum point distance in this segment with respect to the reference contour. Significant defects are detected by thresholding in both length and depth.
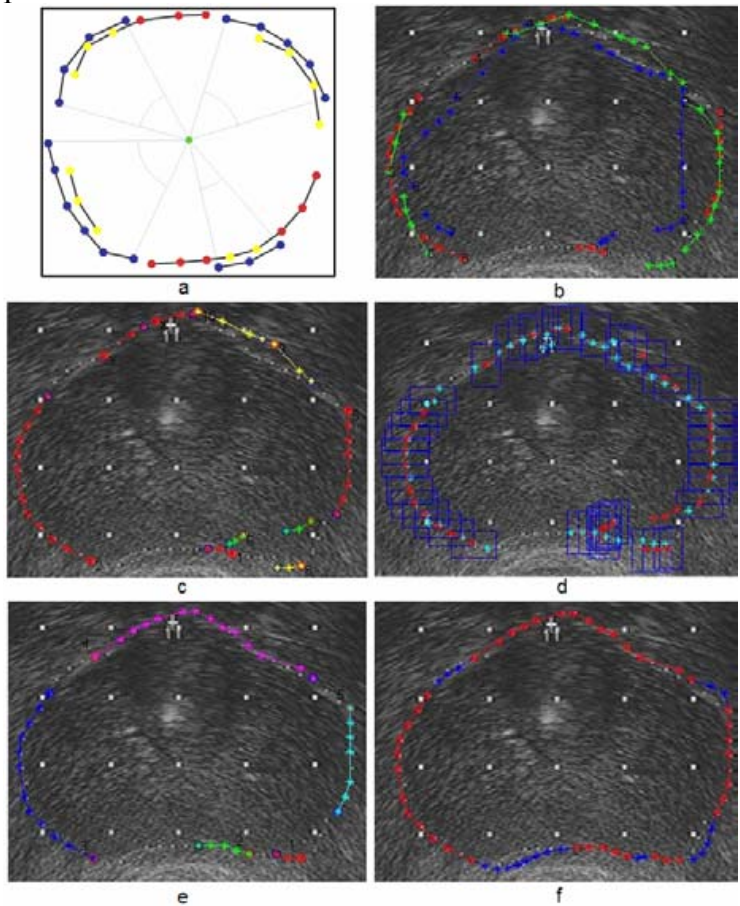

Fig. 5. a) partially overlapping contours; b) superposition of selected chains from slices i-1 (green) and i+1 (blue) on current slice i (red); c) elimination of chain overlap; d) seed-based detection of new points (light blue); e) final set of chains; f) closed prostate contour via Bezier interpolation

Table 1 summarizes the performances of our approach computed over the experimental database. The length and depth thresholds for the selection of significant defects 
were set both to $2 \mathrm{~mm}$. Fig. 7 shows local defects on patient-specific 3D surface models built from series of reference and automatically retrieved contours. A graphical user interface allows for visualizing the correspondence between local maxima in the distance matrix and surface defects. Our experimental results and the defect visualization were considered very promising from a clinical standpoint.

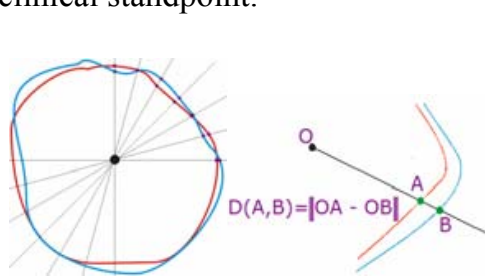

a

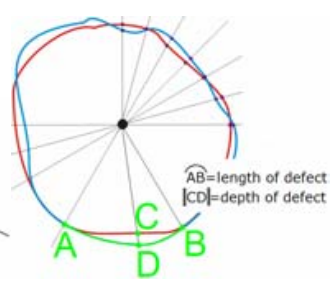

b
Fig. 6. a) Computing distances between pairs of points in reference (red) and automatically retrieved (blue) contours; b) Length and depth of a local defect (green)

\section{CONCLUSIONS}

The proposed approach automatically extracts prostate contours sparsely annotated by radiologists on TRUS images. Our main theoretical contribution consists in the design of a task-oriented, bottom-up method which mimics perceptual grouping mechanisms for extracting virtual contours. The proposed contour retrieval approach embeds anatomic and procedural knowledge.

From a practical standpoint, the proposed approach is of high clinical relevance, since it is applicable to archived databases containing annotations that are blended with the image content. From these databases, contour information can be retrieved with our approach and used for the generation of 3D prostate models for therapy planning and monitoring. Ongoing work focuses on using the 3D prostate models for tracking postinterventional oedema evolution.

Table 1. Statistics on local defects in automatically retrieved contours computed over database with respect to manually segmented contours chosen as reference.

\begin{tabular}{|l|l|l|l|}
\hline $\begin{array}{l}\text { Average no. of } \\
\text { significant } \\
\text { contour defects } \\
\text { per patient }\end{array}$ & $\begin{array}{l}\text { Mean and std. } \\
\text { deviation of } \\
\text { surface overlap }\end{array}$ & $\begin{array}{l}\text { Mean and std. } \\
\text { deviation of } \\
\text { defect length }\end{array}$ & $\begin{array}{l}\text { Mean and std. } \\
\text { deviation of } \\
\text { defect depth } \\
(\mathrm{mm})\end{array}$ \\
\hline $3 \pm 2$ & $98.61 \pm 3 \%$ & $7.76 \pm 7.64 \mathrm{~mm}$ & $3.00 \pm 1.47 \mathrm{~mm}$ \\
\hline
\end{tabular}

\section{REFERENCES}

[1] T. Wittenberg, "The need for annotation for reference image datasets", International Congress Series 1281, pp. 453-458, 2005.
[2] P. Abolmaesumi and M.R. Sirouspour, "Segmentation of prostate contours from ultrasound images", Proc. IEEE Int. Conf. on Acoustics, Speech, and Signal Processing, 2004.

[3] D.Shen, Y.Zhan, and C. Davatzikos, "Segmentation of Prostate Boundaries From Ultrasound Images Using Statistical Shape Model", IEEE Trans. on Med. Imag., 22[4], 2003.

[4] G. Lixin, S.D. Pathak, et al., "Parametric shape modeling using deformable superellipses for prostate segmentation", IEEE Trans. on Med. Imag., 23[3], 340-349, 2004.

[5] Y. Zhan and D. Shen, "Deformable Segmentation of 3-D Ultrasound Prostate Images Using Statistical Texture Matching Method", IEEE Trans. on Med. Imag.,25[3], 256 - 272, 2006.

[6] P.J. Elliott, J. Diedrichsen, K.J. Goodson, R. Riste-Smith, and G.J. Sivewright, "An object-oriented system for 3D medical image analysis", IBM Systems Journal, 35 [1], 1996.

\section{Distance matrix computed for the prostate volume}

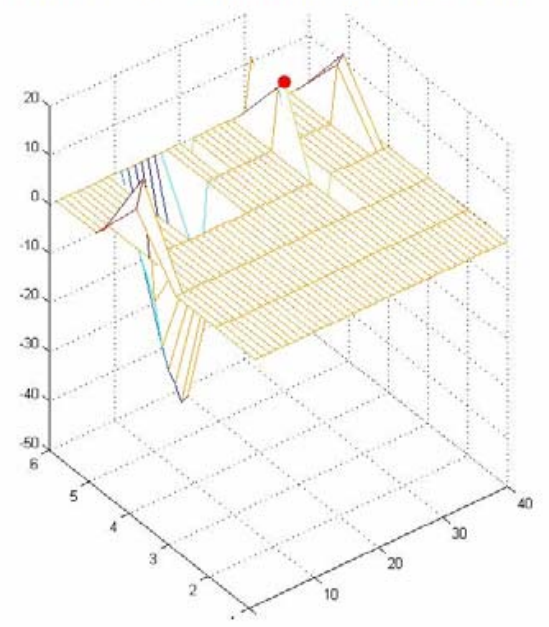

Surface built from reference contours

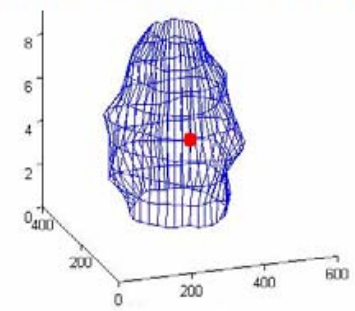

Surface built from automatically retrieved contours

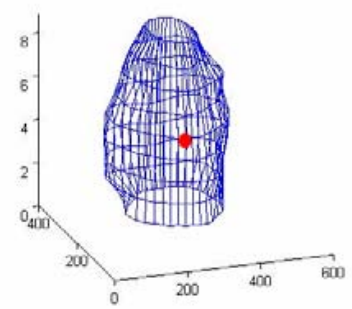

Fig. 7. Interactive visualization of a significant defect (red dot) on patient-specific 3D prostate models and on the distance map 